\title{
Pulsed Ti: Sapphire Laser Power Amplifier
}

\author{
E. R. Ault, G. V. Erbert, B. W. Sleaford, E. L. Pierce, D. W. Crozier
}

February 23, 1999

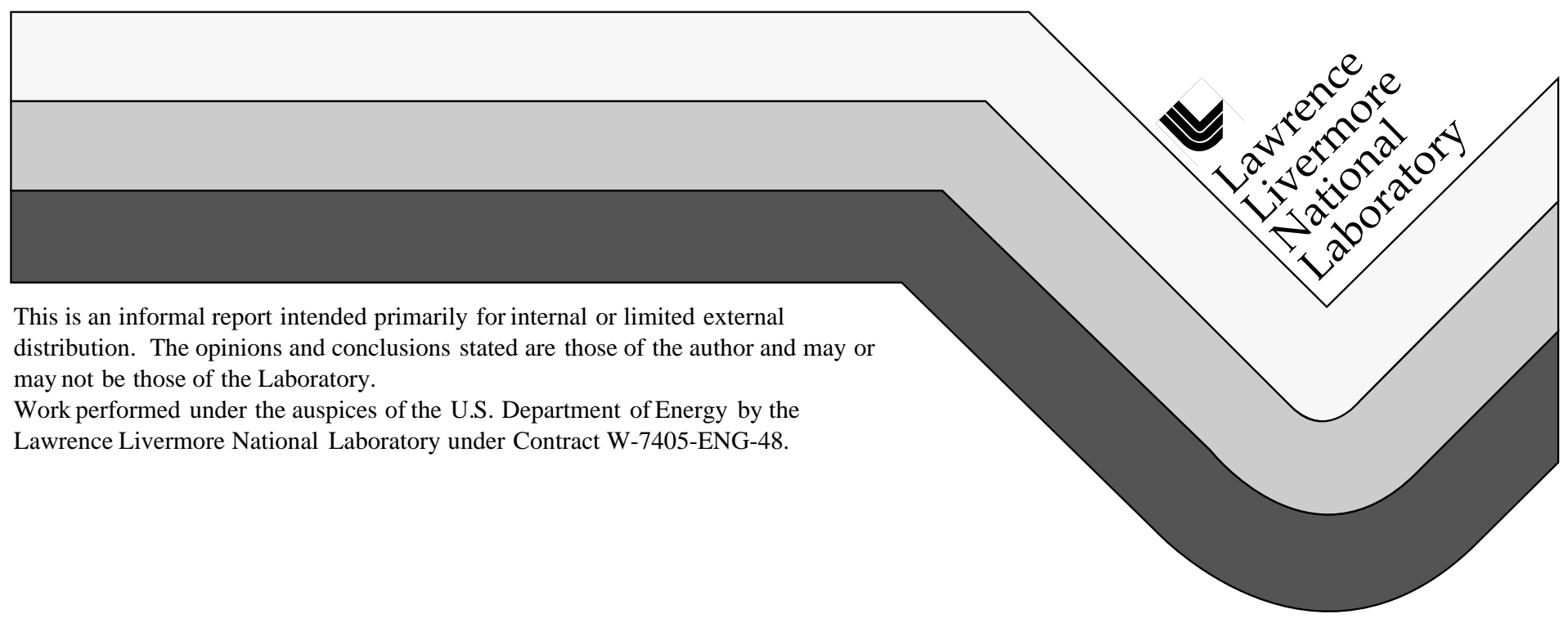




\section{DISCLAIMER}

This document was prepared as an account of work sponsored by an agency of the United States Government. Neither the United States Government nor the University of California nor any of their employees, makes any warranty, express or implied, or assumes any legal liability or responsibility for the accuracy, completeness, or usefulness of any information, apparatus, product, or process disclosed, or represents that its use would not infringe privately owned rights. Reference herein to any specific commercial product, process, or service by trade name, trademark, manufacturer, or otherwise, does not necessarily constitute or imply its endorsement, recommendation, or favoring by the United States Government or the University of California. The views and opinions of authors expressed herein do not necessarily state or reflect those of the United States Government or the University of California, and shall not be used for advertising or product endorsement purposes.

This report has been reproduced directly from the best available copy.

Available to DOE and DOE contractors from the Office of Scientific and Technical Information P.O. Box 62, Oak Ridge, TN 37831

Prices available from (423) 576-8401

Available to the public from the National Technical Information Service

U.S. Department of Commerce 5285 Port Royal Rd. Springfield, VA 22161 
UCRL-ID-133449

February 23, 1999

\title{
98-ERD-069 Final Report \\ Pulsed Ti:Sapphire Laser Power Amplifier
}

\author{
E. R. Ault, G. V. Erbert, B. W. Sleaford, E. L. Pierce and D. W. Crozier
}

\section{Introduction}

The unique thermal properties of Ti:Sapphire at low temperatures allows this laser material to be scaled to high powers while maintaining excellent beam quality. This has been demonstrated with an Argon-ion pumped, continuous wave Ti:Sapphire laser. Ti:Sapphire can also be efficiently pumped with the second harmonic of solid-state Nd:YAG lasers. Recent development of high average power frequency doubled Nd:YAG lasers suggest that Ti:Sapphire could be scaled to higher powers and efficiencies using these lasers as the pump source.

There are also numerous applications based on frequency-doubled, tunable near-infrared light that call for power levels and beam quality presently unavailable. The tunability of Ti:Sapphire could be exploited with the expectation that frequency doubling will give access to the ultraviolet portion of the spectrum. Applications could also require pulsed lasers operating at $10 \mathrm{kHz}$ or higher. Beam quality is essential for achieving (1) efficient propagation over long distances, (2) high brightness for precise focal properties, and (3) efficient frequency doubling. These lasers are also valuable (operating at the fundamental wavelength or frequency doubled) for other materials processing including welding, cutting, and surface treatment of precision parts.

The goal of this project is to demonstrate an all solid-state, high power Ti:Sapphire laser system. The important parameters are beam quality and electrical-to-optical efficiency at high average powers. A complete laser system would consist of a narrow band, low power oscillator followed by several stages of amplification. To investigate high average powers in a simple laser system, a broad band power oscillator followed by a single stage amplifier was constructed. By adjusting the output power of the oscillator, the extraction and efficiency characteristics of each stage of amplification could be studied in a single amplifier. From this information, a complete laser system can be designed and specified.

\section{Hardware}

The solid-state pump lasers used in this work are diode-pumped, frequency doubled Nd:YAG lasers designed and constructed at LLNL. The lasers are acousto-optically Q-switched and the second harmonic is generated with a $5 \times 5 \times 18 \mathrm{~mm}$ Lithium Triborate (LBO) crystal. Output powers up to 120 watts at $532 \mathrm{~nm}$ can be achieved with these lasers operating at pulse repetition rates from 10 to $15 \mathrm{kHz}$. The output beam quality was measured to be between 30 and 50 times diffraction limited. Three of these lasers, one for the oscillator and two for the amplifier, were constructed to support this project. An additional pump laser is currently under construction to increase the total pump power for future experiments. 
The output power of Ti:Sapphire lasers with stable high quality beams is limited by thermal gradients generated in the rod. Beam distortions induced by these gradients can be reduced significantly by cooling the rod to liquid nitrogen temperature. The thermal conductivity of Sapphire increases by a factor of 30 and dn/dT decreases by a factor of 7 when cooling from room temperature to $77^{\circ} \mathrm{K}$. The Ti:Sapphire laser designed and constructed for this work is enclosed in a vacuum vessel to prevent condensation on the rod surfaces when cooled to low temperatures. The Ti:Sapphire rods are mounted to a copper block which is cooled with flowing liquid nitrogen. An optical breadboard inside the vacuum chamber allows the laser to be easily configured to various optical designs.

Figure 1 shows the optical schematic of the power oscillator and single pass amplifier used in these experiments. The resonator is an end pumped, astigmatic compensated z-cavity. The pump beam is introduced into the rod with a $45^{\circ}$ fold mirror with a small hole. The resonator beam passes through the hole in the pump mirror. Because of the relatively large size of the pump, beam less than $2 \%$ of the pump light is lost due to the hole in the mirror. Earlier designs used dichroic mirrors which transmitter the pump beam and reflected the resonator beam. These mirrors would often be damaged due to the high peak power intensity of the resonator beam which could exceed $50 \mathrm{MW} / \mathrm{cm}^{2}$. The light from the power oscillator is focused into the single pass amplifier with a simple lens. The amplifier can be pumped by two pump lasers using the same technique as the oscillator.

\section{Modeling}

An important feature of the laser design is to convert a multimode pump beam into a near diffraction limited output beam. The spatial overlap of the pump and resonator beams within the rod along with the beam diameters are critical design parameters. Modeling using Gaussian beam propagation techniques coupled with a Frantz-Nodvik amplifier model was used to optimize the optical design of laser resonator and amplifier. The efficiency of the oscillator and amplifier is largely determined by the pump beam size in the laser rod. Figure 2 shows the results of a Frantz-Nodvik amplifier model plotting the normalized efficiency versus beam radius in a Ti:Sapphire crystal for three different pump beam qualities. The optimum beam size in the rod increases as the mode quality of the pump beam decreases. For our case, with a pump beam quality of 50 times diffraction limited (XDL), the optimum beam radius is 150 microns.

A simple plano-convex lens is used to focus the pump beam into the rod. Figure 3 shows the results of a Gaussian beam propagation model plotting the beam radius as a function of position in the laser rod for both the pump and signal beams. The Brewster surfaces of the rod generate beams that are astigmatic and anamorphic so both the $\mathrm{X}$ and $\mathrm{Y}$ axes are plotted. The pump lens is tilted at $9.8^{\circ}$ about the $\mathrm{Y}$-axis to correct the astigmatism in the pump beam. In this calculation the signal beam is diffraction limited while the pump beam is $50 \mathrm{XDL}$. Using a $150 \mathrm{~mm}$ focal length lens with a $26 \mathrm{~mm}$ diameter pump beam the average beam waist of the $\mathrm{X}$ and $\mathrm{Y}$ axes is 160 microns. This is a good match to the optimum waist size predicted by Frantz-Nodvik amplifier model. Figure 3 also illustrates the difficulty in achieving a good spatial overlap of the pump and resonator beams within the rod because of the large difference in mode quality. The mode quality of the pump laser ultimately limits the over all efficiency of the laser system. 


\section{Experimental Results}

Figure 4 shows the output power of the oscillator as a function of pump power. The power of the pump laser was controlled by the combination of a half wave plate and polarizer. This permitted the power delivered to the oscillator to be varied without changing the mode quality of the pump beam. The maximum output power of the oscillator was 23 watts at a pump power of 100 watts. The laser operated in a broad band mode centered at $870 \mathrm{~nm}$ due to the reflectivity of the mirrors. The optical-to-optical efficiency was $23 \%$ with an electrical-to-optical efficiency of $1.25 \%$. For comparison, the Argon-ion pumped Ti:Sapphire laser currently has an electrical-to-optical efficiency of .02\%. The spatial mode of the output beam is shown in Figure 5. Although the beam quality was not measured, the mode would break up into a $\mathrm{TEM}_{01}$ mode when the resonator was slightly misaligned suggesting that the laser was operating in the TEMoo mode with proper alignment.

Figure 6 shows the output power of the amplifier as a function of injected signal from the oscillator. The total pump power delivered to the amplifier was 84 watts in this experiment. With 10.9 watts of injected power the output from the amplifier was 23.8 watts. The optical-to-optical efficiency of the amplifier under these conditions was $15.4 \%$. Also shown in Figure 6 is the predicted output power based on the Franz-Nodvik model used in the optical design. The model agrees well with the data at the lower injected power levels but overpredicts the output power at the higher injected powers. One explanation of this is that the mode size and shape of the oscillator could change as the pump power is increased. This in turn could effect the beam size, waist location and alignment through the amplifier. The alignment of the amplifier is optimized at low power before the chamber is pumped down. This allows full access to all of the alignment optics. Only one mirror was motorized to correct any misalignment through the amplifier under vacuum. In the future, we plan to motorize all axes of the focusing lens as well as the mirror so that the alignment can be fully optimized under vacuum.

\section{Summary}

We have demonstrated an all solid state Ti:Sapphire laser system consisting of a power oscillator and single pass amplifier. The electrical-to-optical efficiency far exceeds that of the current $\mathrm{CW}$ systems. The pump lasers have lower capital and operating costs than the Argon-ion laser.

In the future, we plan to scale the output power to higher levels by adding a fourth pump laser and improving the output power of the current pump lasers. Modeling results shown in Figure 2 suggest that a large increase in efficiency can be realized by improving the beam quality of the pump lasers, even at the cost of reduced output power. We will explore this option by adding apertures to the cavity and/or reducing the rod diameter along with optimizing the resonator design. Other improvements in efficiency which will be investigated include double passing the amplifier for better extraction.

To complete this work, the laser system will be converted into a two-stage amplifier. A narrow band, lower power oscillator currently under development will be injected in to the amplifier to study the extraction and efficiency characteristics of the amplifier throughout the tuning range of Ti:Sapphire. Detailed beam quality measurements will also be made. Other work will include doubling the narrow band output for materials processing applications.

AI 99-023/GE:dlb

L-24948-01 


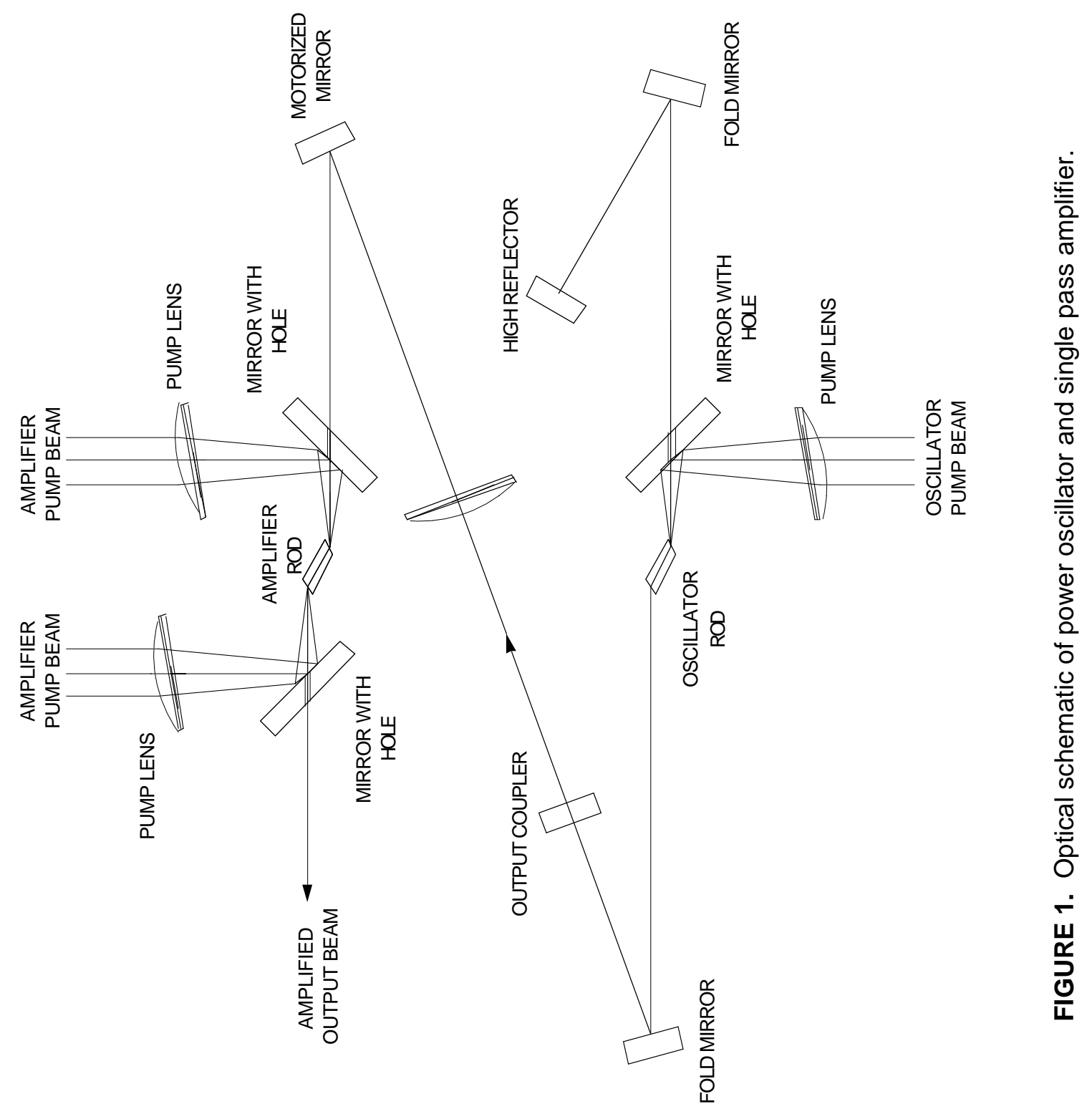



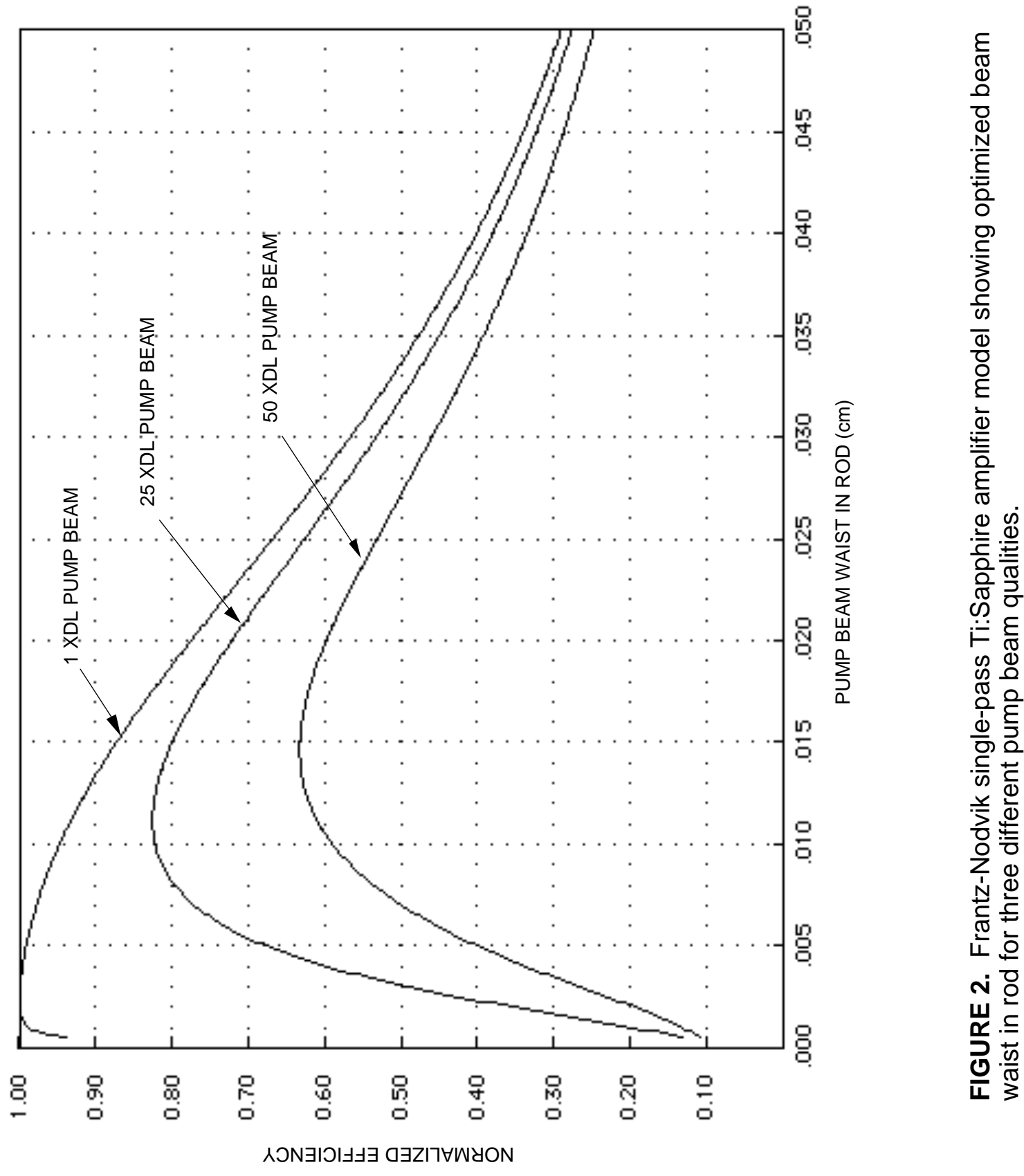


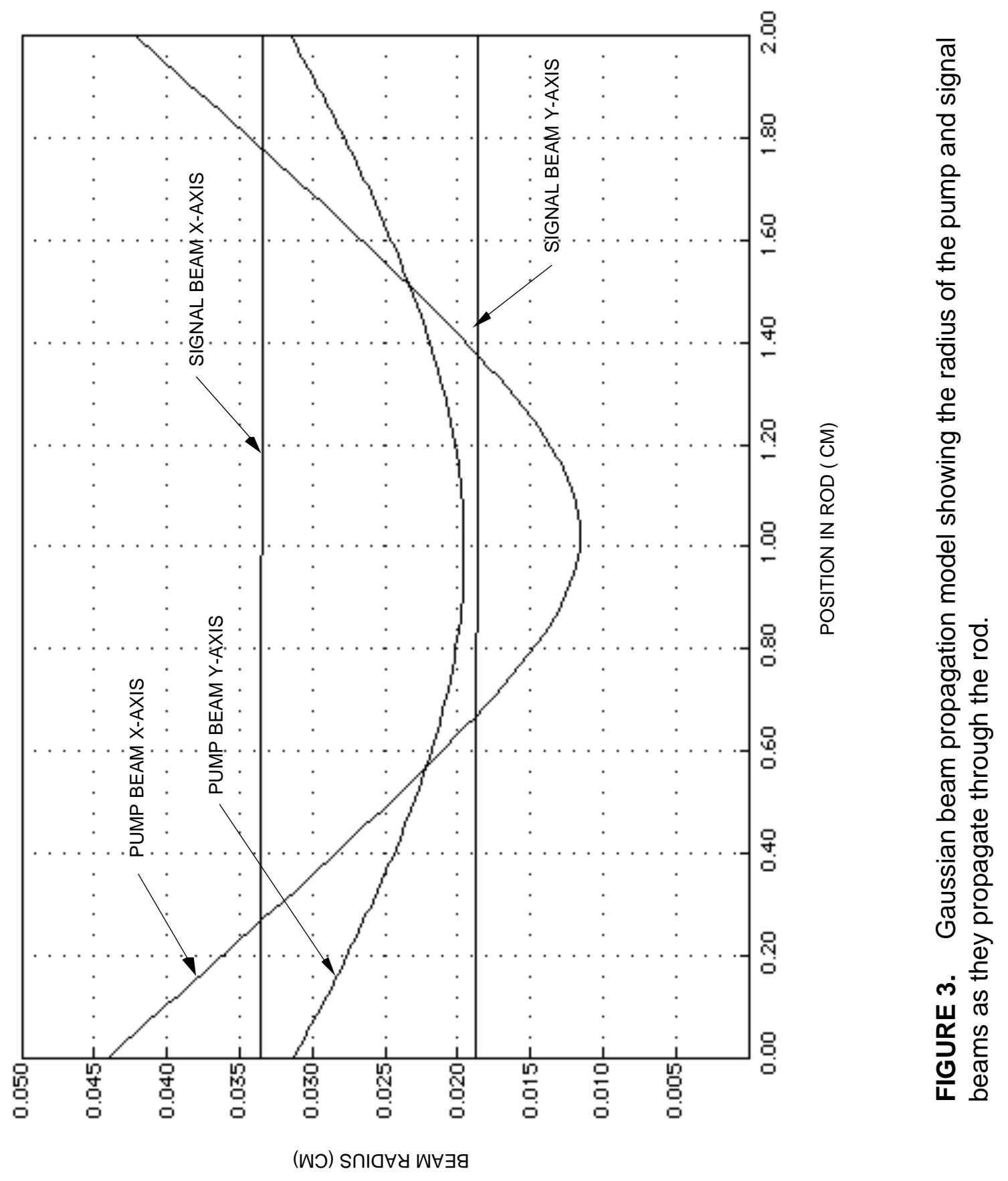




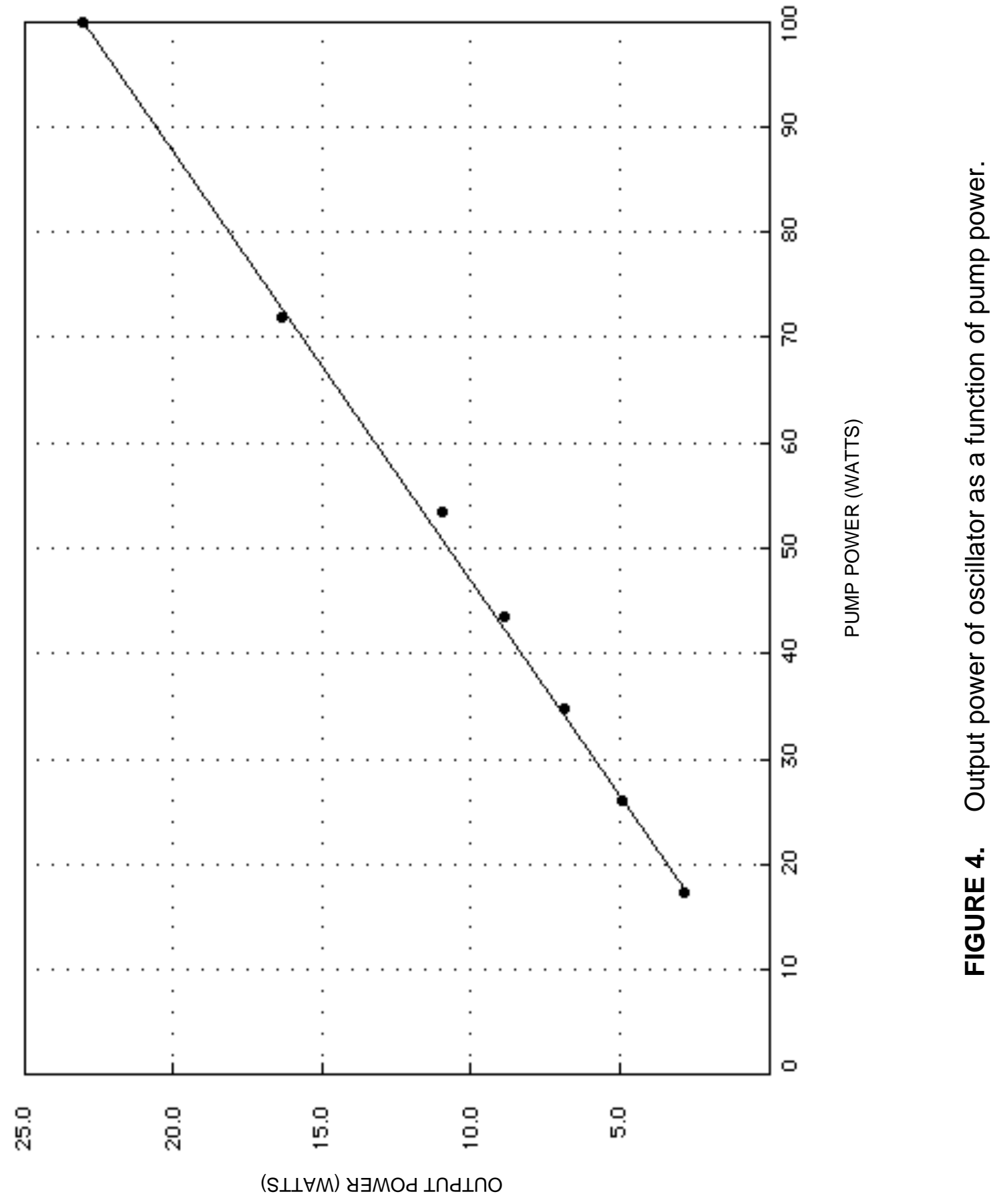




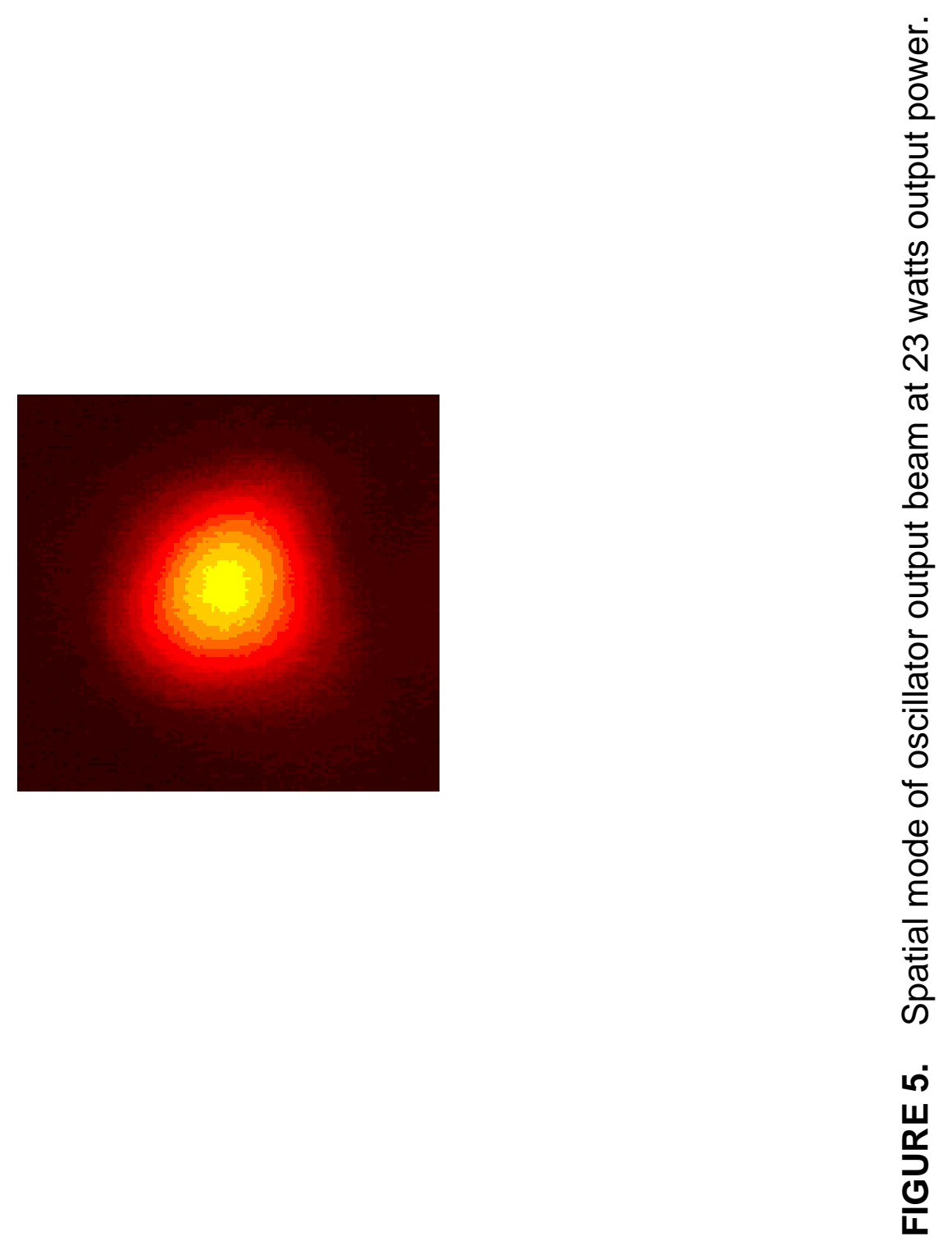




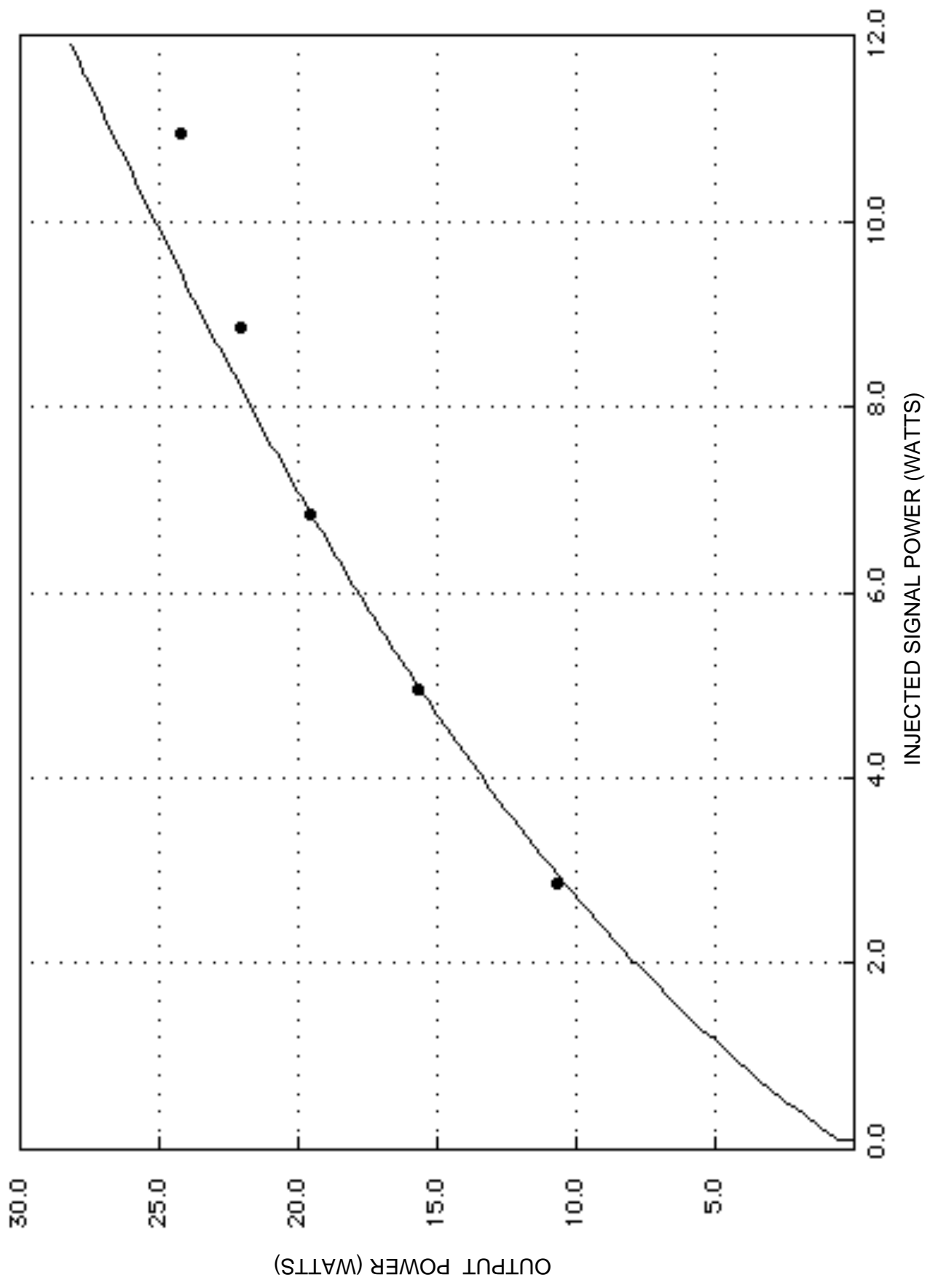

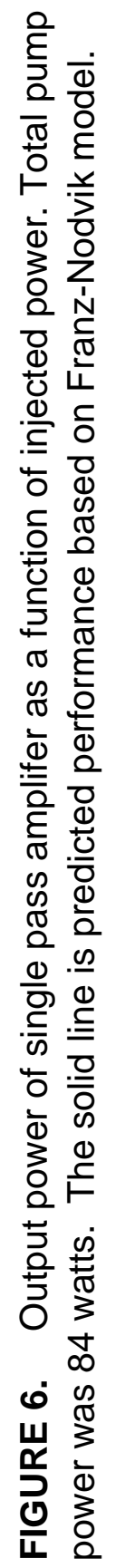

